\title{
Urethral Stone: A Rare Cause of Acute Retention of Urine in Men
}

\author{
Ahmed Ibrahimi*, Idriss Ziani, Jihad Lakssir, Hachem El Sayegh, Lounis Benslimane, \\ Yassine Nouini
}

Department of Urology A, Ibn Sina University Hospital, Faculty of Medicine and Pharmacy, Mohammed V University, Rabat, Morocco

Email: *ahmed.ibrahimi@um5s.net.ma

How to cite this paper: Ibrahimi, A., Ziani, I., Lakssir, J., El Sayegh, H., Benslimane, L. and Nouini, Y. (2020) Urethral Stone: A Rare Cause of Acute Retention of Urine in Men. Open Journal of Urology, 10, 145-151. https://doi.org/10.4236/oju.2020.105016

Received: March 12, 2020

Accepted: April 23, 2020

Published: April 26, 2020

Copyright (c) 2020 by author(s) and Scientific Research Publishing Inc. This work is licensed under the Creative Commons Attribution International License (CC BY 4.0).

http://creativecommons.org/licenses/by/4.0/

(c) (i) Open Access

\begin{abstract}
Urethral stones are a very rare form of urolithiasis, they most often originate from the upper urinary tract or bladder, and are rarely formed primarily in the urethra, it is formed on a urethral anatomical pathology in the majority of cases. The clinical symptomatology is very variable ranging from simple dysuria with penile pain to acute retention of urine. Smaller stones can be expelled spontaneously without intervention, but larger stones or complicated stones or those developed on an underlying urethral anatomical pathology require surgical treatment. The minimally invasive treatment should be the preferred route for the surgical treatment of this disease when feasible. We report the case of a young man with no particular pathological history who presented to the emergency department for acute retention of urine secondary to a primary fossa navicularis calculus, through this case, we discuss the different clinical aspects, etiology, pathogenesis, diagnosis and therapy of urethral stone in men.
\end{abstract}

\section{Keywords}

Urethral Stone, Meatotomy, Acute Urinary Retention, Penis

\section{Introduction}

Urethral stones are very rare localization of urinary stones, and they are in most cases located at the posterior urethra, most often secondary to the migration of bladder stones or upper urinary tract stones, and rarely formed primarily in the urethra, they are relatively more frequent in childhood and rare in females, its clinical manifestations vary widely, ranging from simple and progressive dysuria to acute retention of urine or even more serious complications [1] [2]. 
These stones are generally small and not very symptomatic, they can be single or multiple; however, very large stones are sometimes diagnosed, which represents a challenge for therapeutic management [2].

Their treatment remains poorly codified, it is discussed on a case by case basis and depends upon the form, size and location of the stones, as well as the existence or not of an underlying urethral pathology.

We report the case of a patient admitted to the emergency department for acute retention of urine due to impacted urethral calculus in the fossa navicularis, who benefited from a meatotomy with excellent postoperative results with 4-year follow-up.

\section{Case Presentation}

A 49-year-old man, with no specific medical history, including history of urolithiasis, urethritis or urethral trauma, who had consulted the emergency department for acute retention of urine, penile pain and foreign body sensation in the urethra.

The clinical history of his illness dates back to a year by the sensation of a mobile intraurethral foreign mass, which gradually increased in volume, with dysuria and sensation of incomplete urination which was complicated by an acute retention of urine justifying his consultation in the emergency department.

The physical examination of the penis revealed a hard intraurethral foreign body impacted in the fossa navicularis (Figure 1), the external urethra meatus was normal and the rest of the clinical examination was without abnormality.

The radiography of the pelvis centered on the external genitalia objectified the presence of a calculus at the penile urethra of $5 \mathrm{~cm}$ of the long axis (Figure 2), the radiological exploration of the upper urinary tract showed no other calculus localization. The bladder ultrasound had confirmed the presence of vesical globe with no suspicious bladder lesion or presence of bladder stones, the prostate was of normal size and consistency. The biological examination carried out in emergency did not objectify an anomaly.

After an initial urinary diversion in emergency by a suprapubic catheter, the surgical extraction of this calculus was carried out in a second step after eliminating a urinary tract infection by a small meatotomy because of the size and the distal localization of the calculus, this stone was removed after having blocked it at the distal urethra by applying a tight compress to the level of the root of the penis to prevent any migration into the bladder, the extraction of this stone was carried out by Bengolea forceps while ensuring do not damage the urethral mucosa to avoid any subsequent stenosis or fistula, the meatotomy was then sutured by two separate points of non-absorbable threads and a Foley catheter (18 Fr) was left in place at the end of the intervention (Figures 3-5).

The post-operative follow-ups were simple with the removal of the suprapubic catheter at the end of the intervention, and removal of surgical thread and the urethral catheter twelve days after intervention. After removal of the urethral catheter, the patient resumed normal urination without dysuria or pain. 


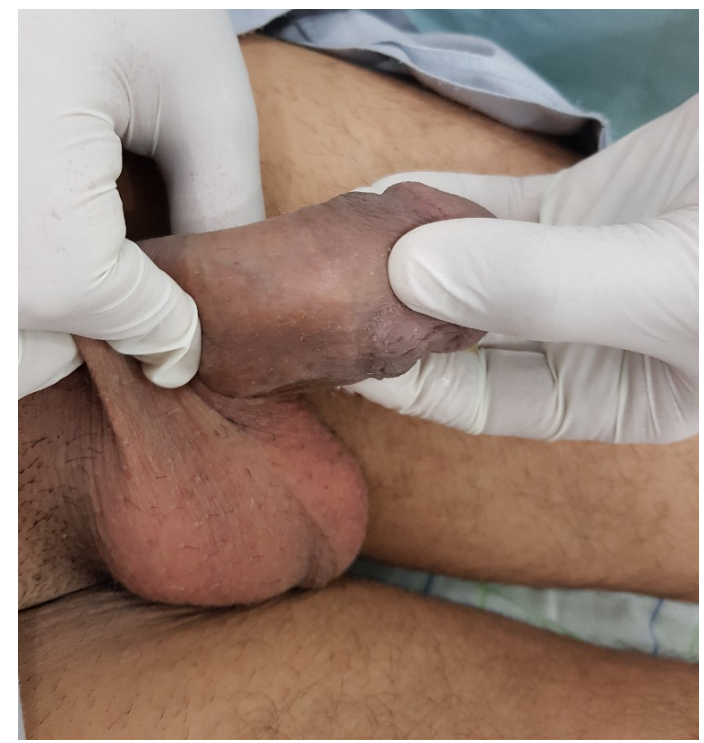

Figure 1. Physical exam showing location of stone.

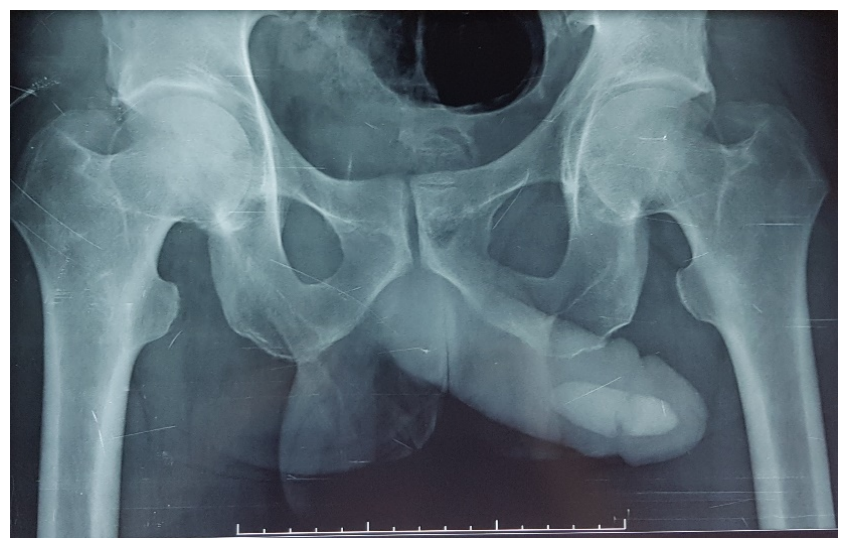

Figure 2. X-ray appearance of a primary fossa navicularis calculus.

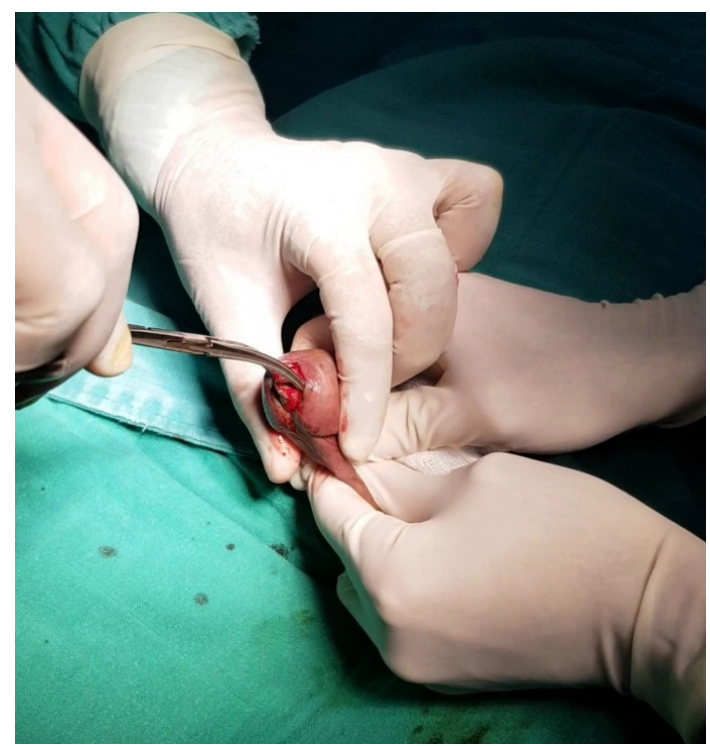

Figure 3. Urethral stone extraction by meatotomy. 


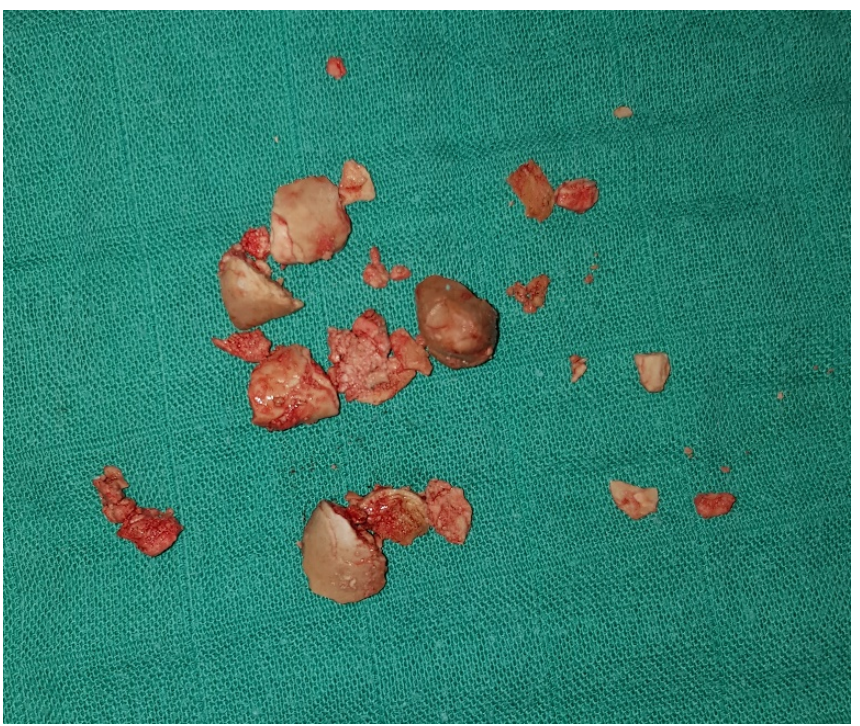

Figure 4. Aspects of extracted urethral stones.

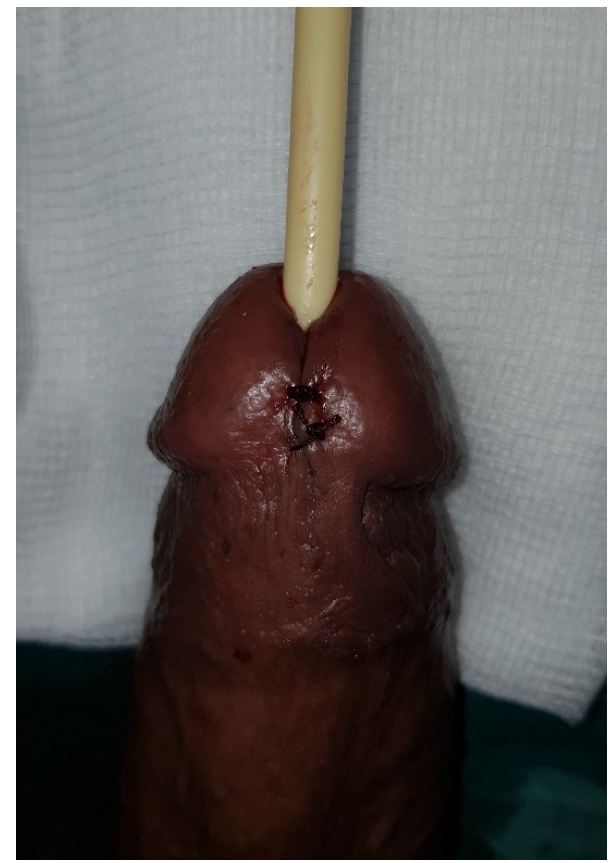

Figure 5. postoperative aspects with urethral catheter.

The results of infrared spectrophotometric analysis of the calculus showed that the stones were composed of ammonium magnesium phosphate.

The evolution with a 4-year follow-up was marked by the resumption of a good urinary stream with normal debimetry, without post-voiding residue and with a normal appearance of the external urethral meatus.

\section{Discussion}

Urethral calculus is a very rare pathological entity, with an incidence lower than $0.3 \%$ of all urinary lithiasis [3]. The frequency of urethral calculi differs accord- 
ing to geographic areas, they are diagnosed individually in Western countries and endemically in the Middle East and Asia [4]. They are most often seen in children because of the high frequency of bladder stones, and rarely observed in women due to the anatomic peculiarities of the female urethra [1]. They are most often secondary to a urethral pathology such as a stenosis of the urethra, urethral diverticulum, hypospadias, tumor of the urethra or a meatal stenosis in young adults and exceptionally developed on a normal urethra, it is preferentially localized at the posterior urethra, and very rarely at the anterior urethra, they can be solitary or multiple [1] [4] [5].

The chemical composition of stones also varies according to geographic areas, in developing countries they generally consist of struvite and uric acid, while in industrialized societies calcium oxalate and cystine are dominant [1]. Calcium oxalate and cystine stones originate mainly from the kidneys, while struvite and uric acid stones originate from the bladder [1].

The clinical symptomatology is very variable ranging from simple penile pain with dysuria to complete urinary retention [6], these untreated or ignored stones may lead to serious complications such as the formation of an abscess and necrosis, urinary fistula or even penile gangrene [2] [6].

The diagnosis is most often easy by interrogation which seeks the history of urinary stones disease or emission of calculus, penile pain, and by the clinical examination which made it possible to palpate a mobile hard urethral mass when it is not impacted and located at the anterior urethra [6]. In other cases, the diagnosis can be confirmed by a simple radiography centered on the external genitalia or a penile ultrasound in the case of radiolucent stones which are not rare [7].

The treatment remains poorly codified, they depend on the size, the number, the localization of the calculus as well as the presence or not of an underlying urethral pathology and the existence or not of a complication modifying the surgical approach [1] [7]. Minimally invasive endoscopic approach by urethroscopy is to be preferred for small proximal calculi with the possibility of extracting them or fragments in situ or pushing them back into the bladder and fragmenting them by laser or lithotripsy with success rates close to 80\% [1] [6] [7]. For a large, multiple, distal stones and stones encrusted in the urethra, conventional open surgery with a meatotomy or sometimes an urethrotomy with or without urethroplasty is an excellent therapeutic means [2] [7].

Regarding open surgical treatment, Mbouché [6] reported the case of three patients with obstructive and impacted urethral calculi successfully treated by an open urethrolithotomy. Agwu [7] also reported in a series of 39 patients the different surgical treatment methods depending on the location and size of the stones, as well as the existence or not of an underlying urethral pathology, a meatotomy was performed in $11(27.5 \%)$ patients, an open urethrolithotomy in $6(15 \%)$ patients, urethrolithotomy and urethroplasty in $2(5 \%)$ patients, this series highlights the essential place that occupies open surgical treatment in the management of distal and impacted urethral calculi. Our choice of open surgical 
procedures as the preferred approach for our patient was thus justified, and supported by the experience and perspective of these authors. Finally, this treatment must be part of a global approach where an etiological treatment must be considered if necessary to prevent recurrence and complications.

Our case highlighted that urethral calculi remains a very rare but not exceptional cause of acute retention of urine in men which remains dominated in the majority of cases by benign prostatic hyperplasia and stenosis of the urethra, our patient consulted immediately for acute retention of urine with impossibility of urethral catheterization because of the size and the distal localization of the calculus, the presence of the calculus of this size impacted at the fossa navicularis poses the problem of its etiology, especially that the exploration of the upper urinary tract did not reveal kidney or ureteral stones, and the lower urinary tract did not reveal a stenosis of the urethra nor bladder or prostatic pathologies or post-voiding residue, the chemical composition of the calculus agrees with its possible renal origin and which has gradually increased in size at the fossa navicularis given its anatomical particularity.

Considering the size and the distal localization of the calculus in the urethra, the open surgery by a simple meatotomy was selected after its discussion with the patient and his agreement, this surgery occupies a very important place particularly in developing countries where access to different endourological techniques is not always possible. Through this case, we hope to draw the attention of practitioners and especially emergency department doctors to this no exceptional etiology, especially in the case of urethral catheterization failure, and that a simple clinical examination and standard imaging will allow the diagnosis to be made in the majority of cases.

\section{Conclusion}

The urethral stone remains a rare cause of acute retention of urine in men, its presence does not exempt from a complete examination of the entire urinary tract in search of a calculus or underlying urethral pathology. The minimally invasive treatment is to be preferred whenever possible, and open surgery retains a major place in the management of large stones impacted or in distal localization.

\section{Conflicts of Interest}

The authors declare no conflicts of interest regarding the publication of this paper.

\section{References}

[1] Kamal, B.A., Anikwe, R.M., Darawani, H., Hashish, M. and Taha, S.A. (2004) Urethral Calculi: Presentation and Management. BJU International, 93, 549-552. https://doi.org/10.1111/j.1464-410X.2003.04660.x

[2] Ramdass, M.J. and Naraynsingh, V. (2014) Multiple Urethral Stones Causing Penile Gangrene. Case Reports in Urology, 2014, Article ID: 182094.

https://doi.org/10.1155/2014/182094 
[3] Safwat, A.S., Hameed, D.A., Elgammal, M.A., Abdelsalam, Y.M. and Abolyosr, A. (2013) Percutaneous Suprapubic Stone Extraction for Posterior Urethral Stones in Children: Efficacy and Safety. Urology, 82, 448-450.

https://doi.org/10.1016/j.urology.2013.03.028

[4] Verit, A., Savas, M., Ciftci, H., Unal, D., Yeni, E. and Kaya, M. (2006) Outcomes of Urethral Calculi Patients in an Endemic Region and an Undiagnosed Primary Fossa Navicularis Calculus. Urological Research, 34, 37-40.

https://doi.org/10.1007/s00240-005-0008-2

[5] Gadimaliyev, E. (2013) Urethral Diverticulum Calculi in a Male: A Case Report. Case Reports in Urology, 2013, Article ID: 437106. https://doi.org/10.1155/2013/437106

[6] Mbouché, L.O., Andzé, G.O., Makon, A.S.N., Bob, D.N., Tamufor, E.N., Amenglé, A.L., et al. (2019) Acute Urinary Retention in the Male Child from Urethral Calculi: A Report of Three Cases. Case Reports in Urology, 2019, Article ID: 5762139. https://doi.org/10.1155/2019/5762139

[7] Agwu, N.P., Abdulwahab-Ahmed, A., Sadiq, A.M., Oyibo, E.U. and Mungadi, I.A. (2020) Management of Impacted Urethral Calculi: An Uncommon Cause of Acute Urine Retention in North-Western Nigeria. International Journal of Clinical Urology, 4, 1 . 